\title{
Increased Prostate Imaging-Reporting and Data System Scores in Multiparametric Magnetic Resonance Imaging May Predict More Extensive Disease in Radical Prostatectomy Specimens
}

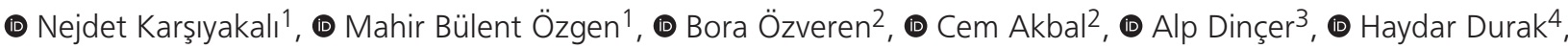 \\ (1) Levent Türkeri ${ }^{1}$ \\ ${ }^{1}$ Acıbadem Mehmet Ali Aydınlar University, Altunizade Hospital, Clinic of Urology, Istanbul, Turkey \\ 2Acıbadem Mehmet Ali Aydınlar University Faculty of Medicine, Altunizade Hospital, Clinic of Urology, Istanbul, Turkey \\ ${ }^{3}$ Acıbadem Mehmet Ali Aydınlar University Faculty of Medicine, Altunizade Hospital, Clinic of Radiology, Istanbul, Turkey \\ ${ }^{4}$ Acıbadem Mehmet Ali Aydınlar University, Altunizade Hospital, Clinic of Clinical Pathology Laboratory, Istanbul, Turkey
}

\begin{abstract}
Objective: This study aimed to investigate the correlation between index lesion prostate imaging-reporting and data system (PI-RADS) version-2 score and histopathological outcomes of prostatectomy specimens.

Materials and Methods: A total of 78 male patients with prostate cancer (PCa) treated with robot-assisted radical prostatectomy between August 2015 and June 2020 were included in this study. In this cohort, suspicious lesions on multiparametric magnetic resonance imaging (mpMRI) were scored according to PI-RADS version-2 criteria. MpMRI-targeted prostate biopsy was performed for all suspicious lesions with a PI-RADS score of $\geq 3$ followed by systematic prostate biopsy. The relationship between index lesion PI-RADS score and histopathological outcomes of prostatectomy specimens were evaluated statistically.

Results: The mean age of the patients was $65.0 \pm 7.0$ years. The distribution of PI-RADS scores of 3, 4, and 5 of the index lesions were 6 (7.7\%), 29 (37.2\%), and $43(55.1 \%)$, respectively. Lower tumor volume and tumor volume ratio were observed in patients with a PI-RADS score of 3 when they were compared with patients with PI-RADS scores of 4 and PI-RADS-5 ( $<<0.001$, for each). No significant correlation was found between index lesion PI-RADS score in mpMRI and clinically significant PCa in prostatectomy specimens $(r<0.200, p>0.05)$. However, a significant correlation was observed between index lesion PI-RADS score and extracapsular extension (ECE), as well as seminal vesicle invasion (SVI) and pT stage ( $r=0.327, p=0.004 ; r=0.276, p=0.014, r=0.348, p=0.002, r e s p e c t i v e l y)$.

Conclusion: Increased index lesion PI-RADS scores were associated with ECE, SVI, higher tumor volume, tumor volume ratio, and pT stages. Increased index lesion PI-RADS score in mpMRI may be helpful in prediction of locally advanced PCa in prostatectomy specimens.

Keywords: Index lesion, multiparametric magnetic resonance imaging, PI-RADS, prostate neoplasms, radical prostatectomy
\end{abstract}

\section{Introduction}

Prostate cancer ( $\mathrm{PCa}$ ) is the second most common cancer in men worldwide with an estimated 1,276,106 new cases and 358,989 deaths (1). Transrectal ultrasound-guided systematic prostate biopsy (SBx) with a minimum of 10-12 cores has been accepted as the standard diagnostic approach for the evaluation of patients who have a clinical suspicion for PCa (2). Recently, multiparametric prostate magnetic resonance imaging (mpMRI), which provides a better depiction of prostate anatomy as well as functional imaging, has been recommended for the evaluation of any patient who has a suspicion of PCa with or without a history of prior biopsy $(2,3,4)$.

The European Society of Urogenital Radiology introduced the prostate imaging-reporting and data system (PI-RADS) version-1 (v1) for standardization of interpretation and reporting of mpMRI in 2012 (5). A Likert-type scoring system is used in PI-RADS to demonstrate the likelihood of the presence of clinically significant (cs) PCa. Several limitations such as suboptimal definition of the exact scoring with relatively higher subjectivity in the evaluation

Cite this article as: Karşıyakalı N, Özgen MB, Özveren B, Akbal C, Dinçer A, Durak H, Türkeri L. Increased Prostate Imaging-Reporting and Data System Scores in Multiparametric Magnetic Resonance Imaging May Predict More Extensive Disease in Radical Prostatectomy Specimens. Bull Urooncol 2021;20(3):147-152

Address for Correspondence: Nejdet Karşıyakalı, Acıbadem Mehmet Ali Aydınlar University, Altunizade Hospital, Clinic of Urology, İstanbul, Turkey Phone: +90 5058652463 E-mail: nejdetkarsiyakali@gmail.com ORCID-ID: orcid.org/0000-0002-0709-0331 Received: 02.09.2020 Accepted: 29.09.2020 
of suspicious lesions in PI-RADSv1 system restricted its use (6). Subsequently, PI-RADS version-2 (v2) was introduced in 2015 and several studies suggested that PI-RADSv2 is more suitable for routine clinical use due to its higher reproducibility and better inter-observer agreement for malignant lesions when compared to PI-RADSv1 $(6,7,8)$.

Although higher detection rates of csPCa in mpMRI-targeted prostate biopsy (TBx) specimens in patients with increased PIRADS scores have been reported in many studies, currently, the exact relationship between PI-RADS scores and pathological outcomes at the final prostatectomy specimens remains unclear $(3,9,10,11,12,13,14)$. Several retrospective studies have investigated this topic with controversial outcomes $(15,16,17)$. In this context, the present study aimed to evaluate the association of PI-RADS scores in mpMRI with the pathological outcomes of prostatectomy specimens.

\section{Materials and Methods}

\section{Study Population}

We retrospectively reviewed medical records of 329 male patients who underwent transperineal prostate needle biopsy in Acıbadem Mehmet Ali Aydinlar University, Altunizade and Kadıköy Hospitals, Clinic of Urology, between August 2015 and June 2020. Patients who underwent transperineal TBx, with concomitant 12-core SBx, diagnosed with $\mathrm{PCa}$ and treated with robot-assisted radical prostatectomy were included in the study. The study was approved by the local Institutional Ethics Committee (IRB No:2021-15/01).

All steps of the study were planned and conducted in accordance with the principles of the Declaration of Helsinki. A written informed consent on admittance to hospital was obtained from all individuals, which permitted the use of respective medical information in clinical studies.

Demographic characteristics, preoperative clinical characteristics, pathological findings of each biopsy type, and prostatectomy specimens were noted in detail for each patient. Patients who had benign tissue in biopsy pathology, underwent SBx only, had metastatic PCa at clinical staging, did not accept surgery or treated with radiotherapy and had missing clinical data were excluded. Finally, a total of 78 male patients who met the selection criteria were included in this study. The PI-RADS score groups (scores of 3,4, and 5) were compared statistically.

\section{mpMRI and Determination of Suspicious Lesions}

All patients were evaluated with 3-T mpMRI (Magnetom Skyra, Siemens Healthineers, Erlangen, Germany) before the prostate biopsy. All mpMRI studies were evaluated by the same dedicated radiologist (A.D.), and all PI-RADSv2 lesions $\geq 3$ were mapped. The border of the prostate and lesions were outlined and saved as a biopsy plan by MIM Symphony Dx ${ }^{\text {TM }}$ Software Inc. version 6.7 (Cleveland, OH, USA). The lesion with the highest PI-RADSV2 score was determined as the index lesion in case of multiple suspicious lesions in mpMRI. If more than one lesion had the same highest PI-RADSv2 score, the lesion with the largest volume calculated by MIM Symphony DxTM software was accepted as the index lesion.
Transperineal mpMRI-targeted and Systematic Prostate Biopsy

All transperineal TBx and SBx procedures were performed under sedoanalgesia in a dorsal lithotomy position. An 18-gauge automatic biopsy gun with a 19-mm sample notch was used in biopsy procedures (Tru-Core ${ }^{\mathrm{TM}}$ II URO Automatic Biopsy Instrument, Argon Medical Devices, Inc., TX, USA). A singledose parenteral antibiotic as prophylaxis was administered to all patients during anesthesia induction (ceftriaxone sodium $1 \mathrm{~g}$ or cefuroxime sodium $1.5 \mathrm{~g}$, intravenous). Moreover, 2-4 samples were taken from each of the suspicious lesions with PI-RADS score of $\geq 3$ using a stepper and template grid as previously reported (18). Additional biopsies were performed if necessary (e.g., for the hypoechoic lesions on ultrasonography). All biopsy samples and whole-mount sections after radical prostatectomy were evaluated by a dedicated uropathologist (H.D.) in accordance with the 2014 International Society of Urologic Pathology (ISUP) criteria (19). csPCa in prostatectomy specimens was defined as the presence of Gleason score (GS) $>6$ or GS 6 disease with tumor volume greater than $0.5 \mathrm{~cm}^{3}(20)$.

\section{Statistical Analysis}

Statistical analysis was performed using the Statistical Package for the Social Sciences version 22.0 software (IBM Corp., Armonk, NY, USA). Shapiro-Wilk test was used to check the normality of data for quantitative variables. Descriptive data were expressed in mean \pm standard deviation and median (interquartile range), or number and frequency. Fisher's exact and Kruskal-Wallis tests were used to determine homogeneity, independence, and differences between related groups as indicated. The posthoc analysis was conducted by Tamhane's test. The correlation coefficient and significance for the relationships between the index lesion PI-RADS scores and various variables in biopsy and prostatectomy specimens were calculated with Spearman's test. A two-sided p-value of $<0.05$ was considered statistically significant.

\section{Results}

The mean age of the patients was $65.0 \pm 7.0$ years, and the median PSA and PSA density were 5.52 (4.20-8.80) ng/mL and $0.13(0.09-0.17) \mathrm{ng} / \mathrm{mL}^{2}$, respectively (Table 1). Preoperative demographic and clinical characteristics of the participants and prostatectomy pathology results are summarized in Table 1.

The distribution of PI-RADS scores of 3, 4, and 5 of the index lesions were $6(7.7 \%), 29(37.2 \%)$, and 43 (55.1\%), respectively. The median number of suspicious lesion detected in mpMRI was $3(2-4)$, and the median number of tumor-positive lesion was 1 (1-2) in TBx. The median number of cores sampled and tumorpositive cores in SBx were 12 (12-12) and 3 (1-5), respectively. PCa was detected in $66(84.6 \%)$ patients by TBx and 68 (87.2\%) patients by SBx. csPCa was detected by TBx and SBx in $57(73.1 \%)$ and $61(78.2 \%)$ patients, respectively.

Increased PSA levels was observed in patients with higher PI-RADS score; however, no significant difference was found between the PSA levels of the patients when they were compared according to index lesion PI-RADS score $(p>0.05)$ (Table 2). A significant difference was noted among the PI-RADS score groups in terms 
of the median number of tumor positive lesions in TBx $(p=0.041)$ (Table 2). In the post-hoc analysis, significant difference was found between PI-RADS-3 and PI-RADS-5 groups in terms of the number of tumor positive lesions in $\operatorname{TBx}(\mathrm{p}=0.047)$. In the posthoc analysis, lower tumor volume and tumor volume ratio were observed in PI-RADS-3 when compared with PI-RADS-4 and PI-RADS-5 groups $(p<0.001$, for each). Higher $p$ T stages were observed with increased PI-RADS score, pT3b disease was only seen in the PI-RADS-5 group, while none of the patients in the PI-RADS-3 group had $\geq$ pT3a disease (Table 2).

No significant correlation was found between index lesion PI-RADS score and PSA and PSA density $(r=0.121, p=0.293$; $r=0.120, p=0.296$, respectively). Moreover, no significant correlation was noted between index lesion PI-RADS score and number of tumor positive lesions in TBx ( $r=0.151, p=0.188)$. Significant linear correlation was observed between index lesion PI-RADS score and csPCa in TBx and SBx ( $r=0.300, p=0.008$; $r=0.249, p=0.030$, respectively) (Table 3 ). Similarly, a significant correlation was observed between index lesion PI-RADS score and extracapsular extension (ECE), as well as seminal vesicle invasion (SVI) and pT stage $(r=0.327, p=0.004 ; r=0.276$, $\mathrm{p}=0.014, \mathrm{r}=0.348, \mathrm{p}=0.002$, respectively). Correlation analysis between the index lesion PI-RADS score and prostate biopsy and prostatectomy pathology are summarized in Table 3.

\section{Discussion}

This study aimed to assess the relationship between index lesion PI-RADS score in mpMRI and various pathological features of prostatectomy specimens in patients who underwent transperineal $\mathrm{TBx}$ and/or SBx and subsequently treated with robot-assisted radical prostatectomy. Based on our study results, increased index lesion PI-RADS scores were associated with higher tumor volume, tumor volume ratio, and $\mathrm{pT}$ stages in prostatectomy specimens. Moreover, the frequency of csPCa in prostatectomy specimens increased in patients who had a higher index lesion PI-RADS score. However, no significant difference was found between the PI-RADS score groups in terms of csPCa, and no correlation was noted between the index lesion PI-RADS score and csPCa in prostatectomy specimens. The lack of significant correlation noted between the index

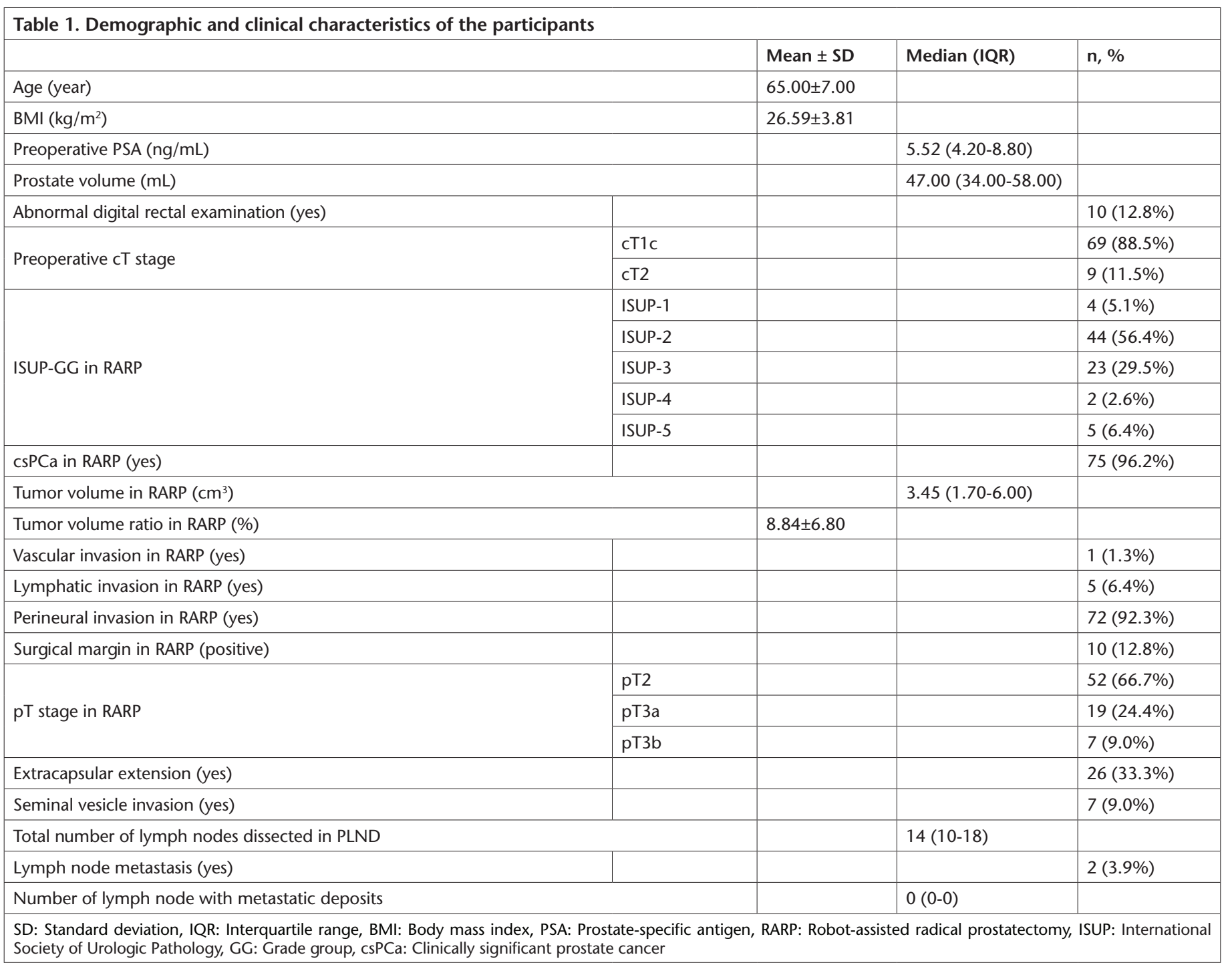




\begin{tabular}{|c|c|c|c|c|c|c|c|c|}
\hline \multirow{3}{*}{\multicolumn{2}{|c|}{ Variables }} & \multicolumn{6}{|c|}{ Index lesion PI-RADS score } & \multirow[b]{3}{*}{ p-value } \\
\hline & & \multicolumn{2}{|c|}{$\begin{array}{c}\text { PI-RADS-3 } \\
(n=6,7.7 \%)\end{array}$} & \multicolumn{2}{|c|}{$\begin{array}{c}\text { PI-RADS-4 } \\
(n=29,37.2 \%)\end{array}$} & \multicolumn{2}{|c|}{$\begin{array}{c}\text { PI-RADS-5 } \\
(n=43,55.1 \%)\end{array}$} & \\
\hline & & median (IQR) & n, \% & median (IQR) & $\mathrm{n}, \%$ & median (IQR) & n, \% & \\
\hline \multicolumn{2}{|c|}{ Abnormal digital rectal examination (yes) } & & 0 & & $3(10.3 \%)$ & & $7(16.3 \%)$ & $\mathrm{a} 0.670$ \\
\hline \multicolumn{2}{|l|}{ Preoperative PSA (ng/mL) } & $\begin{array}{l}4.70 \\
(3.20-4.90)\end{array}$ & & $\begin{array}{l}5.50 \\
(4.07-8.80)\end{array}$ & & \begin{tabular}{|l}
5.72 \\
$(4.40-9.70)$
\end{tabular} & & ${ }^{\mathrm{b}} 0.275$ \\
\hline \multicolumn{2}{|c|}{ Number of positive lesions in TBx } & $0(0-1)$ & & $1(1-2)$ & & $1(1-3)$ & & ${ }^{\mathrm{b}} 0.041^{*}$ \\
\hline \multirow{5}{*}{ ISUP-GG in RARP } & ISUP-1 & & $2(33.3 \%)$ & & $1(3.4 \%)$ & & $1(2.3 \%)$ & \multirow{5}{*}{${ }^{\mathrm{a}} 0.268$} \\
\hline & ISUP-2 & & $3(50.0 \%)$ & & $\begin{array}{l}19 \\
(65.5 \%)\end{array}$ & & $22(51.2 \%)$ & \\
\hline & ISUP-3 & & $1(16.7 \%)$ & & $7(24.1 \%)$ & & $15(34.9 \%)$ & \\
\hline & ISUP-4 & & 0 & & 0 & & $2(4.7 \%)$ & \\
\hline & ISUP-5 & & 0 & & $2(6.9 \%)$ & & $3(7.0 \%)$ & \\
\hline \multicolumn{2}{|l|}{ csPCa in RARP (yes) } & & $5(83.3 \%)$ & & $\begin{array}{l}28 \\
(96.6 \%)\end{array}$ & & $42(97.7 \%)$ & a 0.264 \\
\hline \multicolumn{2}{|l|}{ Tumor volume in RARP $\left(\mathrm{cm}^{3}\right)$} & $\begin{array}{l}0.80 \\
(0.50-1.30) \\
\end{array}$ & & $\begin{array}{l}3.00 \\
(1.70-5.50) \\
\end{array}$ & & $\begin{array}{l}4.50 \\
(2.50-7.30) \\
\end{array}$ & & ${ }^{\mathrm{b}} 0.001^{*}$ \\
\hline \multicolumn{2}{|l|}{ Tumor volume ratio in RARP (\%) } & $\begin{array}{l}1.90 \\
(1.75-2.60)\end{array}$ & & $\begin{array}{l}6.00 \\
(3.10-10.50)\end{array}$ & & $\begin{array}{l}9.30 \\
(6.00-14.50)\end{array}$ & & ${ }^{\mathrm{b}} 0.001^{*}$ \\
\hline \multicolumn{2}{|l|}{ Perineural invasion in RARP (yes) } & & $5(83.3 \%)$ & & $\begin{array}{l}25 \\
(86.2 \%)\end{array}$ & & $42(97.7 \%)$ & a 0.159 \\
\hline \multicolumn{2}{|l|}{ Surgical margin in RARP (positive) } & & 0 & & $5(17.2 \%)$ & & $5(11.6 \%)$ & $\mathrm{a} 0.576$ \\
\hline \multirow{3}{*}{ pT Stage in RARP } & pT2 & & $6(100.0 \%)$ & & $\begin{array}{l}23 \\
(79.3 \%)\end{array}$ & & $23(53.5 \%)$ & \multirow{3}{*}{$\mathrm{a} 0.031^{*}$} \\
\hline & рT3a & & 0 & & $6(20.7 \%)$ & & $13(30.2 \%)$ & \\
\hline & $\mathrm{pT} 3 \mathrm{~b}$ & & 0 & & 0 & & $7(16.3 \%)$ & \\
\hline Extracapsular extension (yes) & & & 0 & & $6(20.7 \%)$ & & $20(46.5 \%)$ & $\mathrm{a} 0.015^{*}$ \\
\hline Seminal vesicle invasion (yes) & & & 0 & & 0 & & $7(16.3 \%)$ & ${ }^{\mathrm{a}} 0.053$ \\
\hline
\end{tabular}

\begin{tabular}{|c|c|c|c|c|c|c|c|c|c|c|c|c|c|c|c|c|}
\hline & \multicolumn{2}{|c|}{ csPCa in TBx } & \multicolumn{2}{|c|}{ csPCa in $\mathrm{SBx}$} & \multicolumn{2}{|c|}{$\begin{array}{l}\text { csPCa in } \\
\text { Prostatectomy }\end{array}$} & \multicolumn{2}{|c|}{$\begin{array}{l}\text { Tumor } \\
\text { volume in } \\
\text { prostatectomy } \\
\left(\mathrm{cm}^{3}\right)\end{array}$} & \multicolumn{2}{|c|}{$\begin{array}{l}\text { Tumor volume } \\
\text { ratio in } \\
\text { prostatectomy } \\
(\%)\end{array}$} & \multicolumn{2}{|c|}{ pT Stage } & \multicolumn{2}{|l|}{ ECE } & \multicolumn{2}{|l|}{ SVI } \\
\hline & $r$ & $p$ & $r$ & $p$ & $r$ & $p$ & $r$ & $p$ & $r$ & $\mathbf{p}$ & $r$ & $p$ & $r$ & $p$ & $r$ & p \\
\hline $\begin{array}{l}\text { Index lesion } \\
\text { PI-RADS }\end{array}$ & $0.300^{* *}$ & 0.008 & $0.249^{*}$ & 0.030 & 0.124 & 0.279 & $0.376^{* *}$ & 0.001 & $0.381^{* *}$ & 0.001 & $0.348^{* *}$ & 0.002 & $0.327^{* *}$ & 0.004 & $0.276^{*}$ & 0.014 \\
\hline
\end{tabular}

lesion PI-RADS score and csPCa in prostatectomy specimens is most likely caused by the very high ratio of csPCa in this cohort. As we presented above, nearly all patients (96.2\%) had csPCa in prostatectomy specimens. By contrast, we observed significant correlation between the index lesion PI-RADS scores and csPCa in both TBx and SBx. Therefore, indirect evidence may suggest that increased index lesion PI-RADS scores may also play a role in predicting csPCa in prostatectomy specimens. Furthermore, higher index lesion PI-RADS scores were correlated with ECE and
SVI in prostatectomy specimens as well as higher volume tumors with higher ISUP-grade group category. In this context, our data may suggest that increased index lesion PI-RADS score in mpMRI may predict locally advanced PCa in prostatectomy specimens.

Currently, mpMRI has been widely accepted as a standard imaging modality in the diagnostic pathway and treatment decision process of PCa with its claimed higher sensitivity for the detection of csPCa $(9,21)$. Previously reported detection rates 
of csPCa for PI-RADS lesions with a score of 3, 4, and 5 ranges from $0 \%$ to $66 \%, 21 \%$ to $98 \%$, and $75 \%$ to $99 \%$, respectively $(9,10,11)$. Our observation for csPCa ratios for index lesions with PI-RADS scores of 4 and 5 in prostatectomy specimens were similar; however, the csPCa ratio for the index lesion with a PI-RADS score of 3 was relatively higher in our study than in other studies. The higher CSPCa ratios for PI-RADS 3 lesions may be attributed to the inclusion of only 6 patients with a PI-RADS score of 3 in our study cohort. In addition, although more than $95 \%$ of our patients had csPCa, these patients with an allocated PI-RADS score of 3 may represent biologically aggressive tumors with deceiving radiological characteristics. An observer bias is also a possibility.

The role of PI-RADS scoring in evaluation of tumor characteristics for clinical decision-making has been a topic of interest more recently. Several studies have reported the effect of PI-RADS vs. scoring on selecting possible candidates for active surveillance (AS) $(22,23,24)$. Woo et al. (23) reported that PI-RADSv2 and PSA density were independent predictors of pathological downgrading in prostatectomy specimens in patients who had GS $7(3+4)$ PCa in the prostate biopsy. In this study, the authors concluded that mpMRI might help identify patients who had an overestimated GS in SBx and assist in selecting potential candidates for AS (23). Similarly, a study reported that combination of PSA density (for threshold $\geq 0.15 \mathrm{ng} / \mathrm{mL}^{2}$ ) and PI-RADS score could help minimize the number of missed csPCa in men with a PI-RADS score of $\leq 3$ (25). In another interesting study, PI-RADSv2 score of $>3$ and front-to-total ratio of periprostatic fat tissue, which was measured in mpMRI and hypothesized as an influencer of the tumor microenvironment by paracrine effect, were independent risk factors for pathologic upgrading in prostatectomy specimens in patients with a biopsy GS $6(3+3)(24)$. In the present study, we observed worse pathological outcomes such as higher frequency of ECE and SVI in prostatectomy specimens in patients with increased PI-RADS scores. Therefore, we also suggest that AS may not be a good treatment choice for patients with high PI-RADS (4 and 5) scores.

Slaoui et al. (15) investigated the correlation between PIRADSv2 score in mpMRI and GS of both prostate biopsies and prostatectomy specimens. In this retrospective study, no significant concordance was found for GS in TBx and prostatectomy specimens according to the index lesion PIRADS score (15). By contrast, similar to our study results, a retrospective study reported a significant association between PI-RADS scores and ECE and large tumor volume in a large number of patients who were eligible for AS (16). In this study, upgrading in prostatectomy specimens and unfavorable prognosis were also demonstrated, and the authors concluded that mpMRI and PI-RADS scoring could be used as a supporting parameter for a more accurate selection of patients eligible for AS (16). Similarly, adverse pathological outcomes such as tumor volume, ECE, lymphovascular invasion, and SVI were also reported in patients with increased PI-RADS scores in the most recent and well-designed multicenter study that investigated the correlation between PI-RADS and histopathological outcomes of prostatectomy specimens (17). Another recent study evaluated the correlation between PI-RADSv2 scores and several outcomes of prostatectomy specimens for central and peripheral tumors separately (26). A poor correlation between PI-RADSv2 score and final GS was reported for both central and peripheral tumors, while a moderate-to-high correlation was reported between PI-RADSv2 score and tumor volume (26). Similar to these studies, in the present study, we observed worse histopathological outcomes such as higher tumor volume, pT stage, ECE, and SVI in prostatectomy specimens in patients with increased index lesion PI-RADS scores.

\section{Study Limitations}

This study has several limitations. First, the retrospective and nonrandomized nature of this study and the relatively small sample size introduce the possibility of selection bias. Second, all PIRADS lesions in mpMRI were interpreted by only one radiologist, and all histopathological specimens were evaluated by only one pathologist. Therefore, we were unable to evaluate the interobserver variability for outcomes. By contrast, we believe that our study results may contribute to the body of knowledge on this subject, in which no consensus has been revealed and has not been well investigated yet. Further investigations with larger cohorts, particularly including more patients with index lesion PIRADS score of 3 , are needed to validate the correlation between index lesion PI-RADS score in mpMRI and histopathological outcomes of prostatectomy specimens.

\section{Conclusion}

In this study, higher tumor volume, tumor volume ratio, and $\mathrm{pT}$ stages in prostatectomy specimens were observed in patients with increased index lesion PI-RADS score in mpMRI. The frequency of csPCa in prostatectomy specimens also increased in these patients. However, no significant difference was found between the PI-RADS score groups in terms of csPCa, and no correlation was observed between the index lesion PI-RADS score and CSPCa in prostatectomy specimens. In addition, higher index lesion PI-RADS scores were correlated with ECE and SVI in prostatectomy specimens. In this context, increased index lesion PI-RADS scores in mpMRI may predict locally advanced $\mathrm{PCa}$ in prostatectomy specimens. Therefore, AS may not be a good treatment choice for patients with increased PI-RADS scores. Further well-designed prospective, randomized studies with larger cohorts are needed to confirm our study results.

\section{Acknowledgements}

Publication: The results of the study were not published in full or in part in form of abstracts.

Contribution: There is not any contributors who may not be listed as authors.

Conflict of Interest: No conflict of interest was declared by the authors.

Financial Disclosure: The authors declared that this study received no financial support.

\section{Ethics}

Ethics Committee Approval: Acıbadem Mehmet Ali Aydınlar University and Acıbadem Healthcare Institutions Medical Research Ethics Committee, IRB No:2021-15/01. 
Informed Consent: A written informed consent on admittance to hospital was obtained from all individuals, which permitted the use of respective medical information in clinical studies.

Peer-review: Externally peer-reviewed.

\section{Authorship Contributions}

Supervision: A.D., H.D., L.T., Concept: B.Ö., C.A., L.T., Design: B.Ö., C.A., L.T., Data Collection or Processing: N.K., Analysis or Interpretation: N.K., M.B.Ö., Literature Search: N.K., M.B.Ö., Writing: N.K., M.B.Ö.

\section{References}

1. Bray F, Ferlay J, Soerjomataram I, et al. Global cancer statistics 2018: GLOBOCAN estimates of incidence and mortality worldwide for 36 cancers in 185 countries. CA Cancer J Clin 2018;68:394-424.

2. Mottet N, van den Bergh RCN, Briers E, et al. EAU - ESTRO - ESUR SIOG Guidelines on Prostate Cancer 2020. European Association of Urology Guidelines. 2020 Edition. Vol presented at the EAU Annual Congress Amsterdam 2020. Arnhem, The Netherlands: European Association of Urology Guidelines Office; 2020.

3. Venderink W, van Luijtelaar A, Bomers JGR, et al. Results of targeted biopsy in men with magnetic resonance imaging lesions classified equivocal, likely or highly likely to be clinically significant prostate cancer. Eur Urol 2018;73:353-360.

4. Choi $\mathrm{YH}$, Kang MY, Sung HH, et al. Comparison of cancer detection rates between trus-guided biopsy and mri-targeted biopsy according to psa level in biopsy-naive patients: a propensity score matching analysis. Clin Genitourin Cancer 2019;17:e19-25. doi: 10.1016/j. clgc.2018.09.007.

5. Barentsz JO, Richenberg J, Clements R, et al. ESUR prostate MR guidelines 2012. Eur Radiol 2012;22:746-757.

6. Tewes S, Mokov N, Hartung D, et al. Standardized Reporting of Prostate MRI: Comparison of the Prostate Imaging Reporting and Data System (PI-RADS) Version 1 and Version 2. PLoS One 2016;11:e0162879. doi: 10.1371/journal.pone.0162879.

7. Weinreb JC, Barentsz JO, Choyke PL, et al. PI-RADS Prostate Imaging Reporting and Data System: 2015, Version 2. Eur Urol 2016;69:16-40.

8. Muller BG, Shih JH, Sankineni S, et al. Prostate cancer: interobserver agreement and accuracy with the revised prostate imaging reporting and data system at multiparametric MR imaging. Radiology 2015;277:741-750.

9. Kasivisvanathan V, Rannikko AS, Borghi M, et al. MRI-Targeted or Standard Biopsy for Prostate-Cancer Diagnosis. N Engl J Med 2018;378:1767-1777.

10. Mertan FV, Greer MD, Shih JH, et al. Prospective evaluation of the prostate imaging reporting and data system version 2 for prostate cancer detection. J Urol 2016;196:690-696.

11. Cash H, Maxeiner A, Stephan C, et al. The detection of significant prostate cancer is correlated with the Prostate Imaging Reporting and Data System (PI-RADS) in MRI/transrectal ultrasound fusion biopsy. World J Urol 2016;34:525-532.

12. van der Leest $M$, Cornel $E$, Israel $B$, et al. Head-to-head comparison of transrectal ultrasound-guided prostate biopsy versus multiparametric prostate resonance imaging with subsequent magnetic resonanceguided biopsy in biopsy-naive men with elevated prostate-specific antigen: a large prospective multicenter clinical study. Eur Urol 2019;75:570-578.

13. Siddiqui MM, Rais-Bahrami S, Turkbey B, et al. Comparison of MR/ ultrasound fusion-guided biopsy with ultrasound-guided biopsy for the diagnosis of prostate cancer. JAMA 2015;313:390-397.

14. Rouviere O, Puech P, Renard-Penna R, et al. Use of prostate systematic and targeted biopsy on the basis of multiparametric MRI in biopsynaive patients (MRI-FIRST): a prospective, multicentre, paired diagnostic study. Lancet Oncol 2019;20:100-109.

15. Slaoui $H$, Neuzillet $Y$, Ghoneim $T$, et al. Gleason score within prostate abnormal areas defined by multiparametric magnetic resonance imaging did not vary according to the PIRADS score. Urol Int 2017;99:156-161.

16. de Cobelli O, Terracciano D, Tagliabue E, et al. Predicting pathological features at radical prostatectomy in patients with prostate cancer eligible for active surveillance by multiparametric magnetic resonance imaging. PLoS One 2015;10:e0139696. doi: 10.1371/ journal.pone.0162879.

17. Kizilay F, Celik S, Sozen S, et al. Correlation of Prostate-Imaging Reporting and Data Scoring System scoring on multiparametric prostate magnetic resonance imaging with histopathological factors in radical prostatectomy material in Turkish prostate cancer patients: a multicenter study of the Urooncology Association. Prostate Int 2020;8:10-15.

18. Özgen MB, Özveren B, Uzel S, et al. Initial outcomes and assessment of the transperineal multiparametric-magnetic resonance imaging/ ultrasonography fusion biopsy method in diagnosing clinicallysignificant prostate cancer. Bull Urooncol 2017;16:42.

19. Epstein JI, Egevad L, Amin MB, et al. The 2014 International Society of Urological Pathology (ISUP) consensus conference on gleason grading of prostatic carcinoma: definition of grading patterns and proposal for a new grading system. Am J Surg Pathol 2016;40:244-452.

20. Epstein JI, Walsh PC, Carmichael M, et al. Pathologic and clinical findings to predict tumor extent of nonpalpable (stage T1c) prostate cancer. JAMA 1994;271:368-374.

21. Ahmed HU, El-Shater Bosaily A, Brown LC, et al. Diagnostic accuracy of multi-parametric MRI and TRUS biopsy in prostate cancer (PROMIS): a paired validating confirmatory study. Lancet 2017;389:815-822.

22. Yim JH, Kim CK, Kim JH. Clinically insignificant prostate cancer suitable for active surveillance according to Prostate Cancer Research International: Active surveillance criteria: Utility of PI-RADS v2. I Magn Reson Imaging 2018;47:1072-9.

23. Woo S, Kim SY, Lee J, et al. PI-RADS version 2 for prediction of pathological downgrading after radical prostatectomy: a preliminary study in patients with biopsy-proven Gleason Score $7(3+4)$ prostate cancer. Eur Radiol 2016;26:3580-7.

24. Zhai L, Fan Y, Sun S, et al. PI-RADS v2 and periprostatic fat measured on multiparametric magnetic resonance imaging can predict upgrading in radical prostatectomy pathology amongst patients with biopsy Gleason score $3+3$ prostate cancer. Scand J Urol 2018;52:333-339.

25. Stevens E, Truong M, Bullen JA, et al. Clinical utility of PSAD combined with PI-RADS category for the detection of clinically significant prostate cancer. Urol Oncol 2020;38:846.e9-16. doi: 10.1016/j. urolonc.2020.05.024.

26. Gundogdu E, Emekli E, Kebapci M. Evaluation of relationships between the final Gleason score, PI-RADS v2 score, ADC value, PSA level, and tumor diameter in patients that underwent radical prostatectomy due to prostate cancer. Radiol Med 2020;125:827-837. 